\title{
Steering Zitterbewegung in driven Dirac systems: From persistent modes to echoes
}

\author{
Phillipp Reck $\odot$, Cosimo Gorini $\odot$, and Klaus Richter ${ }^{*}$ \\ Institut für Theoretische Physik, Universität Regensburg, 93040 Regensburg, Germany
}

(Received 23 October 2019; revised manuscript received 20 February 2020; accepted 21 February 2020; published 13 March 2020)

\begin{abstract}
Although Zitterbewegung - the jittery motion of relativistic particles-was known since 1930 and was predicted in solid-state systems long ago, it has been directly measured so far only in so-called quantum simulators, i.e., quantum systems under strong control, such as trapped ions and Bose-Einstein condensates. A reason for the lack of further experimental evidence is the transient nature of wave-packet Zitterbewegung. Here, we study how the jittery motion can be manipulated in Dirac systems via time-dependent potentials with the goal of slowing down/preventing its decay or of generating its revival. For the harmonic driving of a mass term, we find persistent Zitterbewegung modes in pristine, i.e., scattering free, systems. Furthermore, an effective time-reversal protocol—-the "Dirac quantum time mirror"-is shown to retrieve Zitterbewegung through echoes.
\end{abstract}

DOI: 10.1103/PhysRevB.101.094306

\section{INTRODUCTION}

Zitterbewegung (ZB), i.e., the trembling motion of relativistic particles described by the Dirac equation, was found by Schrödinger already in 1930 [1,2]. The jittery movement is due to the fact that the velocity operator does not commute with the Hamiltonian, and, therefore, it is not a constant of motion. Indeed, the superposition of particle- and antiparticlelike solutions of the Dirac equation leads to harmonic oscillations with, in the case of electrons and positrons, frequency $f=2 m c^{2} / h \sim 10^{20} \mathrm{~Hz}$ and amplitude given by the Compton wavelength $\lambda_{C} \sim 10^{-13} \mathrm{~m}$, whose direct measurement is still beyond experimental capabilities [3].

On the other hand, the requirements for the $\mathrm{ZB}$ are not unique to the relativistic Dirac equation but can, in principle, be fulfilled in any two- (or multi-) band system. Examples thereof are solid-state systems with spin-orbit coupling as proposed by Schliemann et al. in III-V semiconductor quantum wells $[4,5]$ where the energy spectrum is formally similar to the Dirac Hamiltonian. In a solid-state system, the ZB is directly induced by the periodic underlying lattice [6]. The ZB in systems with low-energy effective Dirac-like dispersion was later proposed for carbon nanotubes [7], graphene [8,9], and topological insulators $[10,11]$. Recently, the ZB was further predicted for magnons [12] and exciton-polaritons [13]. $\mathrm{ZB}$ signatures can also be found in the presence of magnetic fields, e.g., in graphene $[9,14]$ and III-V semiconductor quantum wells with spin-orbit coupling [15].

The first experimental observations of the $\mathrm{ZB}$ were achieved with a single ${ }^{40} \mathrm{Ca}^{+}$ion in a linear Paul trap [16] and for Bose-Einstein condensates [17,18] with an induced spin-orbit coupling using atom-light interactions [19].

Recently, indirect experimental realizations of the $\mathrm{ZB}$ in solid-state systems were also reported [20,21]. Also motivated

*klaus.richter@physik.uni-regensburg.de by these, we study time-dependent protocols aimed at prolonging the $\mathrm{ZB}$ duration or at generating revivals by effectively time reversing its decay. On the theory side, in one case, graphene in an external monochromatic electromagnetic field was considered, and a multimode $\mathrm{ZB}$, i.e., a $\mathrm{ZB}$ with additional emerging frequencies, was obtained but found to decay over time [22]. Another work suggests that time-dependent Rashba spin-orbit coupling in a two-dimensional electron gas might indefinitely sustain the ZB [23], whereas adaptation of the two-photon echo method [24], already employed to detect Bloch oscillations [25-27], was also suggested as a tool to create and measure the ZB in carbon nanotubes [28].

Our goal is twofold: (i) To identify nondecaying ZB modes in driven Dirac systems, e.g., graphene; (ii) to consider the possibility of generating ZB "echoes" exploiting the timemirror protocols put forward in Refs. [29,30].

We start in Sec. II with a succinct introduction to the ZB in a static Dirac system, highlighting the mechanisms leading to ZB decay and laying out our general strategy to counteract it via different drivings. In Sec. III, we show that a monochromatic time modulation of the mass term yields a multimode $\mathrm{ZB}$ with more frequencies as compared to driving from a monochromatic electromagnetic field where most importantly additional modes turn out to be long lived. Section IV deals with the generation of ZB echoes/revivals, which, instead, require a short mass gap pulse. Section $\mathrm{V}$ concludes.

\section{ZITTERBEWEGUNG IN DIRAC SYSTEMS: FREQUENCY, AMPLITUDES, AND DECAY}

Consider the static Dirac Hamiltonian,

$$
H_{0}=\hbar v_{F} \mathbf{k} \cdot \boldsymbol{\sigma}+M_{0} \sigma_{z} .
$$

Its eigenenergies are

$$
\varepsilon_{ \pm}(\mathbf{k})= \pm \sqrt{M_{0}^{2}+\hbar^{2} v_{F}^{2}|\mathbf{k}|^{2}}= \pm M_{0} \sqrt{1+\kappa^{2}},
$$


where

$$
\kappa=\hbar v_{F}|\mathbf{k}| / M_{0} .
$$

The eigenstates are

$$
\left|\varphi_{\mathbf{k}, \pm}\right\rangle=\frac{1}{\sqrt{2} \sqrt{1+\kappa^{2} \pm \sqrt{1+\kappa^{2}}}}\left(\begin{array}{c}
1 \pm \sqrt{1+\kappa^{2}} \\
\kappa e^{i \gamma_{\mathbf{k}}}
\end{array}\right)|\mathbf{k}\rangle
$$

with $\gamma_{\mathbf{k}}$ being the azimuthal angle of $\mathbf{k}$ measured from the $k_{x}$ axis.

Considering an initial plane wave with wave-vector $\mathbf{k}$ living in the two bands,

$$
\left|\psi_{0, \mathbf{k}}\right\rangle=c_{\mathbf{k}}^{+}\left|\varphi_{\mathbf{k},+}\right\rangle+c_{\mathbf{k}}^{-}\left|\varphi_{\mathbf{k},-}\right\rangle,
$$

its time evolution is trivially given by

$$
\left|\psi_{\mathbf{k}}(t)\right\rangle=c_{\mathbf{k}}^{+} e^{-i \omega_{\mathbf{k},+} t}\left|\varphi_{\mathbf{k},+}\right\rangle+c_{\mathbf{k}}^{-} e^{-i \omega_{\mathbf{k},-} t}\left|\varphi_{\mathbf{k},-}\right\rangle,
$$

with eigenfrequencies $\hbar \omega_{\mathbf{k}, \pm}=\varepsilon_{ \pm}(\mathbf{k})$. The $\mathrm{ZB}$ is generated by the interference term in the time-dependent expectation value of the velocity operator,

$$
\left\langle\mathbf{v}^{\mathrm{ZB}}\right\rangle_{\mathbf{k}}(t)=2 \operatorname{Re}\left\{c_{\mathbf{k}}^{+}\left(c_{\mathbf{k}}^{-}\right)^{*} e^{-i \Omega_{\mathbf{k}}^{\mathrm{st} t}}\left\langle\varphi_{\mathbf{k},-}|\mathbf{v}| \varphi_{\mathbf{k},+}\right\rangle\right\} .
$$

Here, $\mathbf{v}=\nabla_{\mathbf{k}} H_{0} / \hbar=v_{F} \boldsymbol{\sigma}$ is the velocity operator, and the ZB frequency is given by

$$
\hbar \Omega_{\mathbf{k}}^{\mathrm{st}}=\varepsilon_{+}(\mathbf{k})-\varepsilon_{-}(\mathbf{k})=2 M_{0} \sqrt{1+\kappa^{2}},
$$

where "st" stands for static. Evaluating the matrix element of the velocity operator for the gapped Dirac system yields both parallel and perpendicular ZBs with amplitudes,

$$
\begin{gathered}
A_{\mathbf{k}}^{\mathrm{st}, \|}=v_{F} \frac{2\left|c_{\mathbf{k}}^{+}\right|\left|c_{\mathbf{k}}^{-}\right|}{\sqrt{1+\kappa^{2}}} \leqslant \frac{v_{F}}{\sqrt{1+\kappa^{2}}}, \\
A_{\mathbf{k}}^{\mathrm{st}, \perp}=v_{F} 2\left|c_{\mathbf{k}}^{+}\right|\left|c_{\mathbf{k}}^{-}\right| \leqslant v_{F} .
\end{gathered}
$$

In the perfect (scattering-free) system described by $H_{0}$, the $\mathrm{ZB}$ of a single $\mathbf{k}$ mode oscillates without decaying with an amplitude and frequency given by the initial band-structure occupation. On the contrary, the ZB of a wave packet has a transient character [31], i.e., it vanishes over time. For an initial wave packet of the general form

$$
\left|\psi_{0}\right\rangle=\int d^{2} k \psi_{0}(\mathbf{k})\left|\psi_{0, \mathbf{k}}\right\rangle,
$$

one has

$$
\left\langle\mathbf{v}^{\mathrm{ZB}}\right\rangle(t)=\int d^{2} k\left|\psi_{0}(\mathbf{k})\right|^{2}\left\langle\mathbf{v}^{\mathrm{ZB}}\right\rangle_{\mathbf{k}}(t),
$$

i.e., the wave-packet $\mathrm{ZB}$ is the average of the plane-wave $\mathrm{ZB}$ weighted by the $\mathbf{k}$-space distribution of the initial state. As different $\mathbf{k}$ modes have different frequencies, such a collective ZB dephases over time and vanishes. Technically, this is due to the phase $e^{-i \Omega_{\mathbf{k}}^{\mathrm{st} t}}$ in Eq. (7), whose oscillations as a function of $\mathbf{k}$ become faster for increasing time $t$-and, thus, average progressively to zero. Rusin and Zawadzki give an alternative but equivalent explanation for the ZB decay [32]. They start by considering the movement of the two subwave packets in the different \pm bands, each made up of modes with velocities,

$$
\mathbf{v}_{ \pm}=\nabla_{\mathbf{k}} \varepsilon_{ \pm}(\mathbf{k}) / \hbar .
$$

Since $\mathbf{v}_{+}$is antiparallel to $\mathbf{v}_{-}$, the subpackets move away from each other and progressively decrease their mutual overlap, which translates to a decrease in the interference and, thus, in the ZB [33].

Note that the general velocity of Eq. (13) leads also to other unintuitive effects in solids, e.g., Bloch oscillations. There, a constant electric field leads to oscillations of electrons. The reason is that due to the electric field, the momentum of the electrons changes linearly. Due to a periodic band structure in momentum space, this change leads to an oscillatory behavior of the electrons. As opposed to the ZB, Bloch oscillations have been detected experimentally [25-27].

This paper is devoted to circumventing or reverting the decay of the $\mathrm{ZB}$ via a time modulation of the mass term $M_{0} \rightarrow M_{0}+M(t)$. More precisely, we consider the general time-dependent Hamiltonian,

$$
H=\hbar v_{F} \mathbf{k} \cdot \sigma+M_{0} \sigma_{z}+M(t) \sigma_{z}=H_{0}+H_{1}(t),
$$

and study two scenarios. The first one is based on harmonic (monochromatic) driving, to be dealt with in Sec. III. Here, we follow the strategy of Ref. [22], determining analytically the emerging frequencies of the driven $\mathrm{ZB}$ in our system via the rotating-wave approximation (RWA) and the high-driving frequency (HDF) limit. Our analytics are then compared to numerical simulations based on the "TIME-DEPENDENT QUANTUM TRANSPORT" (TQT) software package [34], which also allows us to study the multimode $\mathrm{ZB}$ in regimes not accessible analytically. By taking a Fourier transform of the numerically obtained time-dependent velocity, we can identify the oscillation frequencies and aim at finding out long-lived or possibly nondecaying modes-i.e., we are after oscillations which survive on a long timescale. To single out the long-time oscillations, we Fourier transform the simulation signal for $t>t^{*}$, where $t^{*}$ is the time when the amplitude of the initial transient oscillations has decayed below $5 \%$ of its $T=0$ value, see Sec. III C.

The second scenario, discussed in Sec. IV, is radically different: Rather than looking for long-lived modes in response to a persistent monochromatic driving, we consider the effects of a sudden (nonadiabatic) on-and-off modulation of the gap. The idea is to use the quantum time mirror (QTM) protocol of Refs. [29,30] to effectively time reverse the sub-wavepacket dynamics. Once the latter are brought back together, we expect a reconstruction of the interference pattern yielding a ZB.

\section{DRIVEN ZITTERBEWEGUNG IN DIRAC SYSTEMS: EMERGENCE OF PERSISTENT MULTIMODES}

We start from Eq. (14) with a harmonically oscillating mass term of the form

$$
M(t)=\tilde{M} \cos \left(\omega_{D} t\right),
$$

and study the resulting $\mathrm{ZB}$, i.e., we time evolve a given initial state according to

$$
i \hbar \frac{\partial}{\partial t} \psi(t)=H(t) \psi(t),
$$

and calculate the expectation value of the velocity operator. 


\section{A. Driven Zitterbewegung: Analytics}

In the following analytical section, we adapt a procedure from Ref. [22], and details of the derivation can be found in Ref. [35].

\section{RWA}

The RWA is well known and often used in quantum optics to simplify the treatment of the interaction between atoms, i.e., few-level systems and a laser field. Although, here, we consider two bands, the system is effectively a two-level system for any arbitrary $\mathbf{k}$ as long as $\mathbf{k}$ is conserved, i.e., for homogeneous pulses. The conditions for the applicability of the RWA are as follows: (i) The amplitude of the time-dependent part $\tilde{M}$ has to be small compared to other internal energy scales of the system; (ii) its frequency is in resonance with one of the level spacings $\omega_{D} \approx \Omega_{\mathbf{k}}^{\mathrm{st}}$. In that case, all high-frequency terms in the Hamiltonian average out at physical timescales, and only the resonant terms survive [36]. Calculations are performed for a single $\mathbf{k}$ mode since the collective wave-packet $\mathrm{ZB}$ is given by the weighted superposition from Eq. (12).

The goal is to solve the time-dependent Dirac equation (14), i.e., to find for all $\mathbf{k}$ the time-dependent occupations of its two nonperturbed bands, given certain initial conditions. One first looks for a SU(2) (pseudo)spin rotation which diagonalizes the time-dependent part of the Hamiltonian. Then, according to the RWA, only slow terms are kept, i.e., outright static ones or those whose time dependence is given by $e^{ \pm i\left(\omega_{D}-\Omega_{\mathbf{k}}^{\mathrm{st}}\right) t}$. Faster terms, in our case, with a time dependence of $e^{ \pm i\left(\omega_{D}+\Omega_{\mathbf{k}}^{\text {st }}\right) t}$ or $e^{ \pm i \omega_{D} t}$, average out rapidly and are dropped. The equations then decouple, yielding a homogeneous second-order differential equation of the harmonicoscillator type. Its time-dependent wave function is then used to compute the expectation value of the velocity operator $\mathbf{v}$. The ZB perpendicular to the propagation direction $\mathbf{k}$ yields

$$
\begin{aligned}
\left\langle v_{\perp}\right\rangle_{\mathbf{k}}= & v_{F} \frac{\hbar \sqrt{1+\kappa^{2}}}{\tilde{M} \kappa}\left\{\left|A_{+}\right|^{2}\left(\Delta+\omega_{R}\right) \sin \left[\left(\omega_{D}+\omega_{R}\right) t\right]\right. \\
& +\left|A_{-}\right|^{2}\left(\Delta-\omega_{R}\right) \sin \left[\left(\omega_{D}-\omega_{R}\right) t\right] \\
& \left.+2 \omega_{R} \operatorname{Im}\left(A_{-}^{*} A_{+} e^{-i \omega_{D} t}\right)\right\}
\end{aligned}
$$

where we define two additional characteristic frequencies,

$$
\begin{gathered}
\Delta=\Omega_{\mathbf{k}}^{\mathrm{st}}-\omega_{D}, \\
\omega_{R}=\sqrt{\Delta^{2}+\frac{\tilde{M}^{2}}{\hbar^{2}} \frac{\kappa^{2}}{1+\kappa^{2}}} .
\end{gathered}
$$

The quantities $A_{ \pm}$are given by the initial conditions (occupation of the two bands) as shown in Appendix A.

The perpendicular $\mathrm{ZB}$ oscillates with three distinct frequencies: $\omega_{D}, \omega_{D} \pm \omega_{R}$. This is in contrast to the standard electromagnetic driving scenario where only the two frequencies $\omega_{D} \pm \omega_{R}$ are obtained [22]. Crucially, the additional $\omega_{D}$ mode turns out to be nondecaying and, thus, determining the ZB long-time behavior as discussed in Sec. III C. Although not shown here, the static limit can be derived from Eq. (17), e.g., by taking the limit $\tilde{M} \rightarrow 0$.
Similar features arise for the parallel-to-k ZB component,

$$
\begin{aligned}
\left\langle v_{\|}\right\rangle_{\mathbf{k}}= & \frac{\hbar v_{F}}{\tilde{M} \kappa}\left\{\left|A_{+}\right|^{2}\left(\Delta+\omega_{R}\right) \cos \left[\left(\omega_{D}+\omega_{R}\right) t\right]\right. \\
& +\left|A_{-}\right|^{2}\left(\Delta-\omega_{R}\right) \cos \left[\left(\omega_{D}-\omega_{R}\right) t\right] \\
& \left.+2 \Delta \operatorname{Re}\left(A_{+}^{*} A_{-} e^{i \omega_{D} t}\right)\right\} \\
& +4 v_{F} \cos \left(\omega_{R} t\right) \operatorname{Re}\left\{A_{+}^{*} A_{-}\right\}+\text {const. }
\end{aligned}
$$

There are now four different frequencies, $\omega_{D}, \omega_{D} \pm \omega_{R}$, and $\omega_{R}$ as opposed to the single $\omega_{R}$ mode of electromagnetic driving [37]. The $\omega_{D}$ mode is once again responsible for the long-time behavior.

\section{2. $\mathrm{HDF}$}

We now investigate the ZB for HDFs $\hbar \omega_{D} \gg \tilde{M}$, thus, extending the analytically accessible regions. The derivation is similar to the RWA with a different initial $\mathrm{SU}(2)$ transformation [22]. The HDF approximation $e^{ \pm 2 i\left(\tilde{M} / \hbar \omega_{D}\right) \sin \left(\omega_{D} t\right)} \approx 1$ allows for solving the remaining differential equations analytically. One obtains (for the perpendicular ZB)

$$
\begin{aligned}
\left\langle v_{\perp}\right\rangle_{\mathbf{k}}= & -\frac{1}{k}\left(\text { const }+2 \Omega_{\mathbf{k}}^{\mathrm{st}} \operatorname{Im}\left\{B_{+} B_{-}^{*} e^{i \Omega_{\mathbf{k}}^{\mathrm{st}} t}\right\}\right) \\
& -\frac{4 \tilde{M}}{k \hbar \omega_{D}} \frac{M_{0}}{\hbar} \sin \left(\omega_{D} t\right) \operatorname{Re}\left\{B_{+} B_{-}^{*} e^{i \Omega_{\mathbf{k}}^{\mathrm{st} t}}\right\},
\end{aligned}
$$

and (for the parallel ZB)

$$
\begin{aligned}
\left\langle v_{\|}\right\rangle_{\mathbf{k}}= & -\frac{1}{k}\left(\text { const }+4 \frac{M_{0}}{\hbar} \operatorname{Re}\left\{B_{+} B_{-}^{*} e^{i \Omega_{\mathbf{k}}^{\mathrm{st} t}}\right\}\right) \\
& +\frac{2 \tilde{M}}{k \hbar \omega_{D}} \Omega_{\mathbf{k}}^{\mathrm{st}} \sin \left(\omega_{D} t\right) \operatorname{Im}\left\{B_{+} B_{-}^{*} e^{i \Omega_{\mathbf{k}}^{\mathrm{st} t}}\right\},
\end{aligned}
$$

where the quantities $B_{ \pm}$are again given by the initial conditions, see Appendix A. Both $\left\langle v_{\perp}\right\rangle_{\mathbf{k}}$ and $\left\langle v_{\|}\right\rangle_{\mathbf{k}}$ have an $\Omega_{\mathbf{k}}^{\text {st }}$ mode as in the static case as well as weaker $O\left(\tilde{M} / \hbar \omega_{D}\right)$ oscillations at $\omega_{D} \pm \Omega_{\mathbf{k}}^{\text {st }}$. Their suppression, going along with the survival of the $\Omega_{\mathbf{k}}^{\mathrm{st}}$ mode, has a simple physical reason: Electrons cannot respond to a driving much faster than the frequencies of their intrinsic dynamics and, therefore, oscillate at $\Omega_{\mathbf{k}}^{\text {st }}$ as if no extra field was present.

\section{B. Driven Zitterbewegung: Numerics}

We test our RWA and HDF analytical results against numerical simulations based on the TQT wave-packet propagation package [34], which uses the Lanczos method [38] to evaluate the action of the time-evolution operator on a wave packet to time evolve an initial state numerically.

In the following, the initial state is a Gaussian wave packet:

$$
\psi_{0}(\mathbf{k})=\frac{1}{\sqrt{\pi \Delta k^{2}}} \exp \left(-\frac{\left(\mathbf{k}-\mathbf{k}_{0}\right)^{2}}{2 \Delta k^{2}}\right),
$$

that equally occupies both bands and which is time evolved in the presence of the time-dependent mass potential. If not otherwise specified, we take $M_{0}$ as the energy unit, choose the center wave-vector $\mathbf{k}_{0}$ such that $\hbar v_{F} k_{0}=0.4 M_{0}$, and a $\mathbf{k}$ space width of $\Delta k=\left|\mathbf{k}_{0}\right| / 10$, where $k_{0}=\left|\mathbf{k}_{0}\right|$. The velocity expectation value is obtained by numerically computing the 

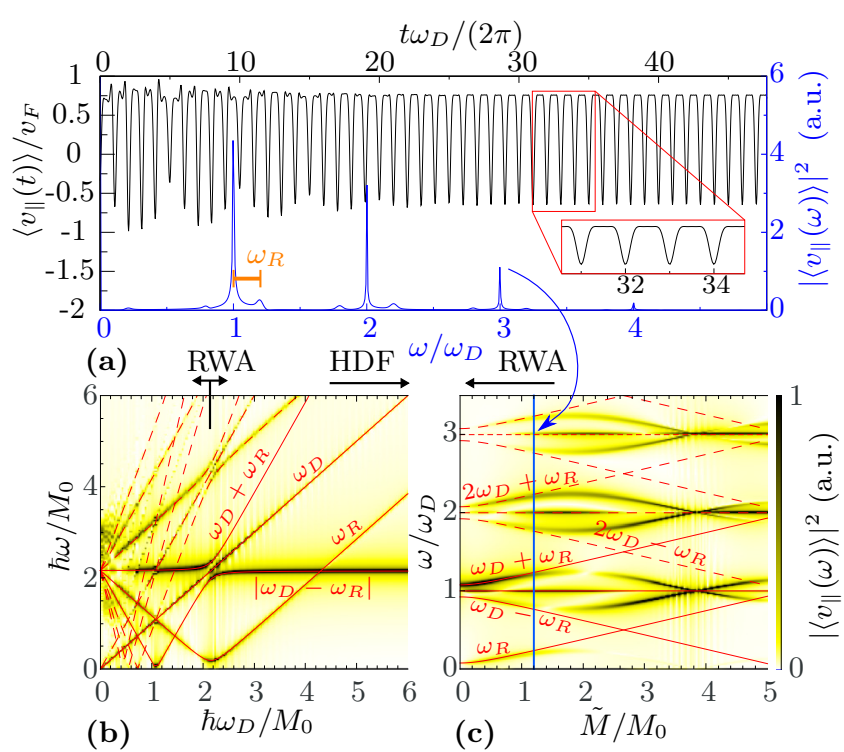

(b)

(c)

FIG. 1. Frequencies of a driven ZB. (a) The parallel ZB $\left\langle v_{\|}(t)\right\rangle$ is shown both as a function of time (black) and as its Fourier transform (blue), i.e., as function of $\omega$ for $\omega_{D}=2 M_{0} / \hbar$ and $\tilde{M}=1.2 M_{0}$. The inset shows a closeup for longer times. Peaks appear in the Fourier transform at multiple integers of $\omega_{D}$ with satellite peaks at a distance of $\omega_{R}$ (orange line at the first peak) away from the major peaks. (b) and (c) Fourier transform of $\left\langle v_{\|}\right\rangle$as a function of driving frequency $\omega_{D}$ (for fixed $\tilde{M}=0.5 M_{0}$ ) and amplitude $\tilde{M}$ (for fixed $\left.\omega_{D}=2 M_{0} / \hbar\right)$ of the time-dependent mass term, respectively. The blue vertical line in (c) marks the function shown in panel (a) (indicated by the blue arrow). The expected frequencies from the RWA $\omega_{D}, \omega_{D} \pm \omega_{R}$, and $\omega_{R}$ are shown (red, solid line) as well as higher-order terms in $\omega_{D}$ (red, dashed line). For smaller $\tilde{M}$, up to $\tilde{M} \approx 1.3 M_{0}$, the RWA results Eq. (20) are well recovered whereas for larger $\tilde{M}$, the RWA is not justified anymore. In all plots, we choose $\mathbf{k}_{0}$ such that $\varepsilon_{+}\left(\mathbf{k}_{0}\right)=0.4 M_{0}$, and, thus, the ZB frequency corresponding to the static $H_{0}$ is $\Omega_{\mathbf{k}_{0}}^{\text {st }} \approx 2.15 M_{0} / \hbar$.

time derivative of the wave-packet position expectation value,

$$
\left\langle\mathbf{v}\left(t_{i}\right)\right\rangle=\frac{\left\langle\mathbf{r}\left(t_{i+1}\right)\right\rangle-\left\langle\mathbf{r}\left(t_{i}\right)\right\rangle}{\delta t},
$$

at each time $t_{i}$ with $\delta t$ as the simulation time step. The static $\mathrm{ZB}$ frequency is $\Omega_{\mathbf{k}_{0}}^{\mathrm{st}} \approx 2.15 M_{0} / \hbar$.

The upper trace in Fig. 1(a) shows the simulation data for $\left\langle v_{\|}\right\rangle$as a function of time (black) for large-amplitude driving $\tilde{M}=1.2 M_{0}$ at frequency $\omega_{D}=2 M_{0} / \hbar$. After an initial irregular transient $\left(t \omega_{D} / 2 \pi \lesssim 20\right)$, a stable periodic signal settles. Note, however, that the long-time response is not monochromatic as manifest from the zoom-in inset. We discuss this in detail below in Sec. III C.

The numerically computed fast Fourier transform of $\left\langle v_{\|}(t)\right\rangle$ is shown in Fig. 1(a) (blue). Clear peaks are visible at integer multiples $\omega=n \omega_{D}, n \in \mathbb{N}$ accompanied by smaller satellite peaks at $n \omega_{D} \pm \omega_{R}$. To visualize the dependence on the different parameters, the fast Fourier transform $\left|\left\langle v_{\|}(\omega)\right\rangle\right|^{2}$ is shown in density plots, each vertical slice corresponding to one simulation at a given value of the driving frequency $\omega_{D}$ for a fixed amplitude $\tilde{M}=0.5 M_{0}$ [panel (b)] and at a given value of the driving amplitude $\tilde{M}$ for a fixed frequency $\omega_{D}=2 M_{0} / \hbar$ [panel (c)]. The vertical blue line in panel (c)
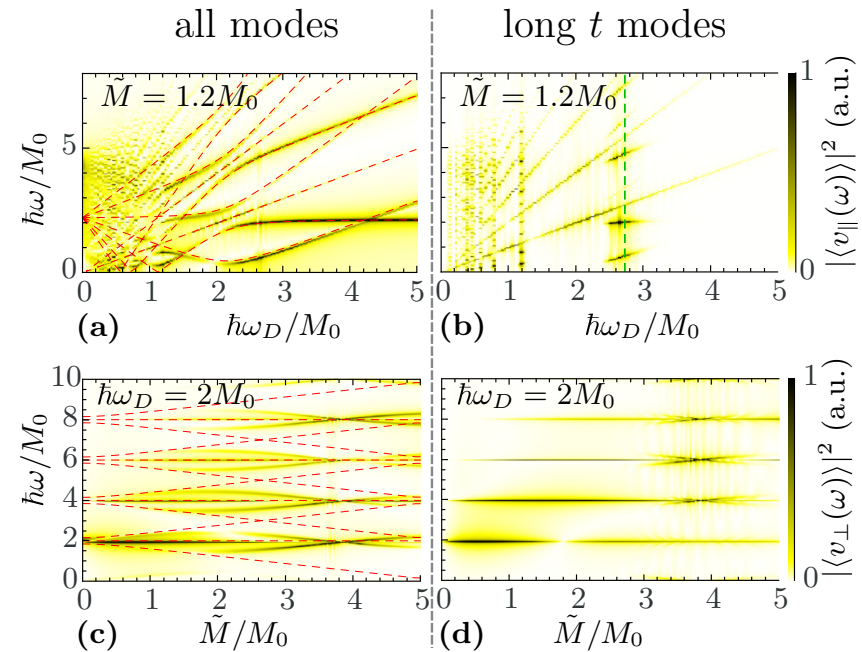

FIG. 2. Searching for infinitely long surviving modes of the Zitterbewegung. The left panels (a) and (c) show plots of all appearing ZB modes whereas the right panels (b) and (d) show the modes which survive a long time (as defined in the text). The modes of the perpendicular and parallel ZBs $\left\langle v_{\perp}(\omega)\right\rangle$ (upper panels) and $\left\langle v_{\|}(\omega)\right\rangle$ (lower panels) are shown as a function either of the parameter $\omega_{D}$ or of $\tilde{M}$. Analytically expected modes from the RWA (red, dashed lines) are indicated in the left panels. In general, the surviving modes for long times are the ones not depending on $\mathbf{k}$, e.g., multiples of $\omega_{D}$, or weakly $\mathbf{k}$ dependent where $\partial \omega_{R} / \partial k=0$ [indicated by the green dashed lines in (b)].

corresponds to the simulation shown in panel (a) as indicated by the blue arrow. The $\mathrm{ZB}$ behaves as expected from the HDF Eq. (22) for large $\omega_{D}$ (only mode: $\Omega_{\mathbf{k}_{0}}^{\text {st }}$ ). Furthermore, in the region of validity of the RWA, the frequencies $\omega_{D}, \omega_{D} \pm \omega_{R}$, and $\omega_{R}$ are present [solid red lines in panels (b) and (c)] as long as $\tilde{M} \lesssim M_{0}$ and $\omega_{D} \approx \Omega_{\mathbf{k}_{0}}^{\text {st }}$. Note that, for $\hbar \omega_{D} \gg \tilde{M}$, one has $\omega_{R} \stackrel{\omega_{D} \rightarrow \infty}{\longrightarrow} \omega_{D}-\Omega_{\mathbf{k}_{0}}^{\text {st }}$ so that one of the RWA modes becomes $\left|\omega_{D}-\omega_{R}\right| \stackrel{\omega_{D} \rightarrow \infty}{\longrightarrow} \Omega_{\mathbf{k}_{0}}^{\text {st }}$ and coincides with the HDF result. Indeed, the line labeled $\left|\omega_{D}-\omega_{R}\right|$ in panel (b) merges with the RWA modes in the region $\omega_{D} \approx \Omega_{\mathbf{k}_{0}}^{\text {st }}$.

In addition to the expected frequencies, further modes also emerge, obtained by adding integer multiples of $\omega_{D}$ to the lower ones. The reason for the appearance of the latter is given in Appendix B, based on higher-order time-dependent perturbation theory.

\section{Long-time behavior of the Zitterbewegung}

We now search for long-lived ZB modes. If present, they should easier to be detected experimentally. The long-time ZB frequencies are easily obtained from the simulation data: By considering the Fourier transform of the signal starting at a time $t>t^{*}$ after the initial transient has decayed. The time $t^{*}$ is defined by the condition that the relative $\mathrm{ZB}$ amplitude in the time-independent setup has decreased to less than $5 \%$ in both $\left\langle v_{\|}\right\rangle$and $\left\langle v_{\perp}\right\rangle$ (if both are present).

In Fig. 2, the ZB frequency spectra for a Fourier transform starting at $t=0$ (left panels) and for a Fourier transform starting at $t^{*}$ (right panels) are compared for several parameter combinations. The simulation data are the same on both sides, 
only the time interval for the fast Fourier transform changes: $\left[0, t_{\max }\right]$ to identify all modes or $\left[t^{*}, t_{\max }\right]$ to single out the long-lived ones, where $t_{\max }$ is maximal time of the simulation. Comparing the left and right panels in Fig. 2, it is clear that some branches fade out completely, some remain nearly unchanged, and others still survive only in a small parameter regime. This can be understood by recalling the general arguments from Sec. II where the ZB decay was shown to be due to dephasing caused by the varying $\mathrm{ZB}$ frequencies of different $\mathbf{k}$ modes building the propagating wave packet. Therefore, the ZB modes that weakly depend on $\mathbf{k}-$ or are outright $\mathbf{k}$ independent-will not dephase and should, thus, survive.

The RWA and HDF approximations indicate that the only k-independent ZB mode has frequency $\omega_{D}$. Indeed, in all plots, modes with frequencies $\omega_{D}$ and integer multiples thereof are unchanged in the long-time limit. The strangely regular shape of the time line for the long-lived $\mathrm{ZB}$ in the closeup of Fig. 1(a) might be explained by the fact that mostly integer multiples of $\omega_{D}$ survive. This corresponds to a discrete Fourier transform and, thus, to a $\omega_{D}$-periodic behavior in time. These $\mathbf{k}$-independent modes are the only truly infinitely lived ones. However, they might be expected since it is not too surprising that a system driven at $\omega_{D}$ will respond at the same frequency (and at multiples thereof). More interestingly, Fig. 2 (b) shows that sections of certain branches survive even at frequencies which are not integer multiples of $\omega_{D}$. Such modes are locally $\mathbf{k}$ independent, i.e., the ZB frequencies are stationary with respect to changes in $\mathbf{k}$. Using Eq. (19) for the RWA frequency $\omega_{R}$ and setting $\partial \omega_{R} / \partial k \stackrel{!}{=} 0$ yields

$$
\begin{aligned}
& \Delta \frac{\partial \Omega_{\mathbf{k}}^{\mathrm{st}}}{\partial k}+v_{F} \frac{\tilde{M}^{2}}{\hbar} \frac{\kappa}{\left(1+\kappa^{2}\right)^{2}}=0 \\
& \Leftrightarrow-M_{0} \omega_{D}+\Omega_{\mathbf{k}}^{\mathrm{st}} M_{0}+\frac{\tilde{M}^{2}}{2{\sqrt{1+\kappa^{2}}}^{3}}=0 .
\end{aligned}
$$

To analyze the data in Fig. 2(b) where the ZB is shown as a function of $\omega_{D}$, we solve Eq. (25) for $\omega_{D}$,

$$
\omega_{D}^{\text {crit }}=\Omega_{\mathbf{k}}^{\text {st }}\left(1+\frac{\tilde{M}^{2}}{4 M_{0}^{2}} \frac{1}{\left(1+\kappa^{2}\right)^{2}}\right) .
$$

The result is shown as a green dashed line in Fig. 2(b), confirming that the system also responds (persistently) at frequencies not directly related to that of the driving. Different ZB modes depend on $\mathbf{k}$ via $\omega_{R}$, and, indeed, each branch intersecting the green line has long-lived components around the intersection point. The long-lived modes are huddled around the exact value of $\omega_{D}^{\text {crit }} \simeq 2.75 M_{0} / \hbar$ (for the given simulation parameters). Note that additional surviving modes, i.e., (locally) $\mathbf{k}$ independent, appear at low frequencies, e.g., $\omega_{D}=1.2 M_{0} / \hbar$. The latter cannot be explained within the RWA, which loses validity for $\omega_{D}<\Omega_{\mathbf{k}}^{\text {st }}=2.15 M_{0} / \hbar$.

The last kind of modes which survive for longer times can be seen in panels (c) and (d) at $\tilde{M} \approx 4 M_{0}$ where the $n \omega_{D}$ modes are crossed by other modes. However, their frequencies are close to the surviving $n \omega_{D}$ modes, and, therefore, they do not, particularly, alter the long-time behavior, which is why we relocate the numerical discussion of their appearance to Appendix C.

\section{ZITTERBEWEGUNG ECHOES VIA EFFECTIVE TIME REVERSAL}

We now discuss an alternative way to retrieve late-time information of the ZB. Instead of Eq. (15), we consider a short mass pulse of the form

$$
M(t)= \begin{cases}\tilde{M}, & t_{0}<t<t_{0}+\Delta t \\ 0, & \text { otherwise }\end{cases}
$$

The steplike form (27) is chosen for definiteness and mathematical convenience only. Indeed, as long as the mass pulse is diabatically switched on and off, it can act as a QTM, i.e., it can effectively time reverse the dynamics of a wave packet, irrespective of its detailed time profile [30,39]. In general, we expect that effective time reversal will yield an echo of the initial ZB. This is apparent when recalling that a propagating two-band wave packet progressively splits into two subwave packets, each composed of states belonging to one of the two bands, and that the ZB is due to the interference between the subwave packets. As the spatial overlap between the latter decreases, so does their interference, causing the ZB to decay [32]. A properly tuned mass pulse, however, causes the subwave packets to invert their occupation of the two bands (the former electronlike state becomes a holelike state and vice versa) and, hence, inverts their direction of motion. Thus, the subwave packets start to reapproach each other, and, as they recover their initial overlap, the interference pattern yielding a $\mathrm{ZB}$ is reconstructed, see Fig. 3 .

\section{A. ZB echoes: Analytics}

We closely follow Refs. [29,30] and quantify the strength of the $\mathrm{ZB}$ echo by considering the density correlator,

$$
\mathcal{C}(t)=\int d^{2} r|\psi(\mathbf{r}, t)||\psi(\mathbf{r}, 0)|
$$

i.e., the spatial overlap between the initial density and the one at time $t$. This measure is appropriate for wave packets which are initially well localized in space. The mass pulse action on an initial eigenstate is $\mathbf{k}$ conserving (the pulse is homogeneous in space) and can, in general, be expressed as a change in the initial band occupation,

$$
\left|\psi_{\mathbf{k}, s}\right\rangle=B_{s}(\mathbf{k}, \Delta t)\left|\varphi_{\mathbf{k}, s}\right\rangle+A_{s}(\mathbf{k}, \Delta t)\left|\varphi_{\mathbf{k},-s}\right\rangle .
$$

For gapped Dirac systems, the transition amplitude $A_{s}$ is independent of $s, A_{s} \rightarrow A$. One has [30]

$$
A(\mathbf{k}, \Delta t)=\frac{i \kappa \tilde{M}}{\sqrt{M_{0}^{2}+M^{2} \kappa^{2}} \sqrt{1+\kappa^{2}}} \sin \left(\frac{M \Delta t}{\hbar} \sqrt{1+\kappa^{2}}\right),
$$

with $M=M_{0}+\tilde{M}$ and $\kappa$ from Eq. (3). Each k mode contributes to the echo only with its component which switches bands (reverses the velocity) during the mass pulse, i.e., the term proportional to $A$ in Eq. (29). Moreover, the components proportional to $B_{s}$ are irrelevant and will be neglected in the following. Given a general initial wave packet,

$$
\left|\psi_{0}\right\rangle=\sum_{\mathbf{k}} \psi_{0}(\mathbf{k})\left|\psi_{0}^{\mathbf{k}}\right\rangle=\sum_{\mathbf{k}} \psi_{0}(\mathbf{k})\left(c_{\mathbf{k}}^{+}\left|\varphi_{\mathbf{k},+}\right\rangle+c_{\mathbf{k}}^{-}\left|\varphi_{\mathbf{k},-}\right\rangle\right)
$$



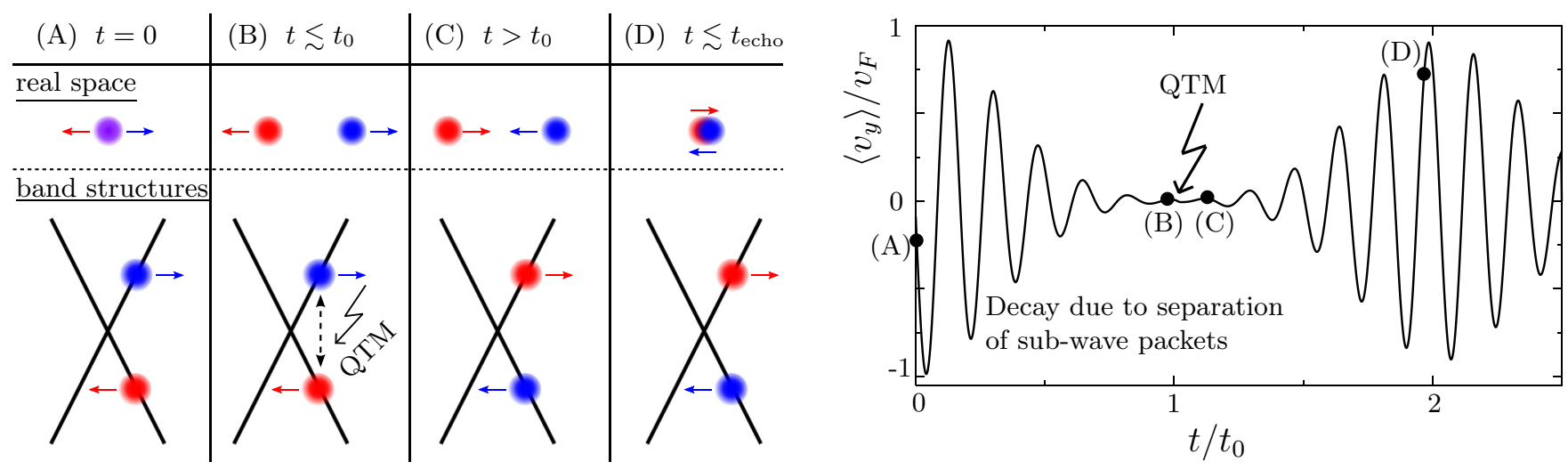

FIG. 3. Retrieving decaying Zitterbewegung through a spin-echo-type time-reversal mechanism. Left panel: An initial wave packet with components in both bands separates due to the different band velocities $\propto \nabla_{\mathbf{k}} \varepsilon(\mathbf{k})$. The decreasing overlap between the subwave packets causes the ZB to decay [(A) to (B)], see the time line of the velocity expectation value $\left\langle v_{y}\right\rangle$ in the right panel. Via a short QTM pulse at $t_{0}$, the subwave packets switch bands, thus, reversing their velocities and returning to their initial positions [see (C)]. The recovery of the overlap in (D) leads to a $\mathrm{ZB}$ revival. The black dots in the right panel belong to the time instants sketched in the left panel.

with $\left|c_{\mathbf{k}}^{+}\right|^{2}+\left|c_{\mathbf{k}}^{-}\right|^{2}=1$, and this amounts to considering only its effectively time-reversed part,

$$
\begin{aligned}
\left|\psi_{\text {echo }}\left(t^{\prime}\right)\right\rangle= & \sum_{\mathbf{k}} \psi_{0}(\mathbf{k})\left|\psi_{\text {echo }}^{\mathbf{k}}\left(t^{\prime}\right)\right\rangle=\sum_{\mathbf{k}} \psi_{0}(\mathbf{k}) \sum_{s= \pm 1} c_{\mathbf{k}}^{s} e^{-i \omega_{\mathbf{k}, s} t_{0}} \\
& \times A(\mathbf{k}, \Delta t) e^{-i \omega_{\mathbf{k},-s} t_{1}}\left|\varphi_{\mathbf{k},-s}\right\rangle
\end{aligned}
$$

Here, $\hbar \omega_{\mathbf{k}, \pm s}=\varepsilon_{ \pm}(\mathbf{k})$, see Eq. (2), whereas $t^{\prime}=t_{0}+\Delta t+t_{1}$ is a generic time after the pulse. For the initial wave packet (31), the $\mathrm{ZB}$ at time $t^{\prime}$ reads

$$
\left\langle\mathbf{v}^{\mathrm{ZB}}\right\rangle\left(t^{\prime}\right)=\int d^{2} k\left|\psi_{0}(\mathbf{k})\right|^{2}\left\langle\mathbf{v}^{\mathrm{ZB}}\right\rangle_{\mathbf{k}}\left(t^{\prime}\right) .
$$

Before the pulse, each $\mathbf{k}$-mode contribution is [see Eq. (7)]

$$
\left\langle v_{i}^{\mathrm{ZB}}\right\rangle_{\mathbf{k}}\left(t<t_{0}\right)=2 \operatorname{Re}\left\{c_{\mathbf{k}}^{+}\left(c_{\mathbf{k}}^{-}\right)^{*} e^{-i \Omega_{\mathbf{k}}^{\mathrm{st} t}}\left\langle\varphi_{\mathbf{k},-}\left|v_{i}\right| \varphi_{\mathbf{k},+}\right\rangle\right\},
$$

where $i \in\{x, y\}$ denotes the direction.

Recall that the wave-packet ZB decay is due to dephasing among its constituent modes: At longer times $t$, the exponential $e^{-i \Omega_{\mathbf{k}}^{\mathrm{st} t}}$ oscillates faster as a function of $\mathbf{k}$ and, thus, averages out in the integral (33). To reverse this dephasing process, the phase of the oscillations must be inverted. For a time $t^{\prime}$ after the pulse, the velocity expectation value of each $\mathbf{k}$ mode of the effectively time-inverted wave-packet $\left|\psi_{\text {echo }}\right\rangle$ in Eq. (32) yields

$$
\left\langle v_{i}^{\mathrm{ZB}}\right\rangle_{\mathbf{k}}\left(t^{\prime}\right)=|A(\mathbf{k})|^{2} 2 \operatorname{Re}\left\{c_{\mathbf{k}}^{+}\left(c_{\mathbf{k}}^{-}\right)^{*} e^{-i \Omega_{\mathbf{k}}^{\mathrm{st}}\left(t_{0}-t_{1}\right)}\left\langle\varphi_{\mathbf{k},+}\left|v_{i}\right| \varphi_{\mathbf{k},-}\right\rangle\right\} .
$$

Indeed, the kinetic phase of the complex exponential $e^{-i \Omega_{\mathbf{k}}^{\text {st }}\left(t_{0}-t_{1}\right)}$ decreases with $t_{1}$ (the time elapsed after the pulse) and reaches zero at $t_{1}=t_{0}-$ i.e., the initial phase is recovered. The recovery happens simultaneously for all $\mathbf{k}$ modes, leading to complete rephasing at the echo time $t_{\text {echo }}=2 t_{0}+\Delta t$ [40]. For a wave packet centered at $\mathbf{k}_{0}$ and narrow enough to approximate $A(\mathbf{k}) \approx A\left(\mathbf{k}_{0}\right)$, the transition amplitude can be taken out of the integral (see Ref. [29]), and the ratio of the revived $\mathrm{ZB}$ amplitude in direction $i, B_{i}^{\text {revival }}$ to the initial one
$B_{i}^{\text {initial }}$ is

$$
\frac{B_{i}^{\text {revival }}}{B_{i}^{\text {initial }}} \approx\left|A\left(\mathbf{k}_{0}\right)\right|^{2},
$$

with $i \in\{x, y\}$. On the other hand, the correlator, Eq. (28), is approximately given by the transition amplitude [29],

$$
\mathcal{C}\left(t_{\text {echo }}\right) \approx\left|A\left(\mathbf{k}_{0}, \Delta t\right)\right|,
$$

so that we expect

$$
\frac{B_{i}^{\text {revival }}}{B_{i}^{\text {initial }}} \approx \mathcal{C}^{2}\left(t_{\text {echo }}\right)
$$

to be checked below numerically.

As a side remark, note that the ZB echo bears a certain resemblance to the spin echo: The latter is achieved when dephased oscillating spins-i.e., an ensemble of two-level systems - are made to rephase again by a $\pi$ pulse. Here, an analogous rephasing among oscillating $\mathbf{k}$ modes-i.e., an ensemble of delocalized states with a given dispersion-is obtained via the QTM protocol.

\section{B. ZB echoes: Numerics}

We numerically compute the correlator $\mathcal{C}(t)$, Eq. (28) via TQT for gapless $\left(M_{0}=0\right)$ and gapped $\left(M_{0} \neq 0\right)$ Dirac systems. In both cases, the transition amplitude is given by Eq. (30). The initial wave packet is composed of $\mathbf{k}$ modes from both branches of the Dirac spectrum, which is necessary for the $\mathrm{ZB}$ at $t=0$. As a proof of principle, we use a Gaussian wave packet, narrow in reciprocal space $\left(\Delta k=k_{0} / 8\right)$, and compare the results with the estimates Eqs. (36)-(38). The wave packet is peaked around $\mathbf{k}_{0}=\left(k_{0}, k_{0}\right)^{T} / \sqrt{2}$ with $\kappa_{0}=$ $\hbar v_{F} k_{0} / M=0.4$.

Figure 4(a) compares the correlator $\mathcal{C}(t)$ to the relative amplitude of the revived ZB. The velocities are normalized with respect to the initial $\mathrm{ZB}$ amplitude $B_{i}^{\text {initial }}$. As expected from the analytics, at the echo time $t_{\text {echo }} \simeq 2 t_{0}$, the $\mathrm{ZB}$ amplitude coincides with the echo strength $\mathcal{C}^{2}\left(t_{\text {echo }}\right) \simeq\left|A\left(\mathbf{k}_{0}, \Delta t\right)\right|^{2}$. Panel (b) shows the relative amplitude both in the $x$ and the $y$ directions, Eq. (36), and echo strengths $\mathcal{C}^{2}\left(t_{\text {echo }}\right)$ as 

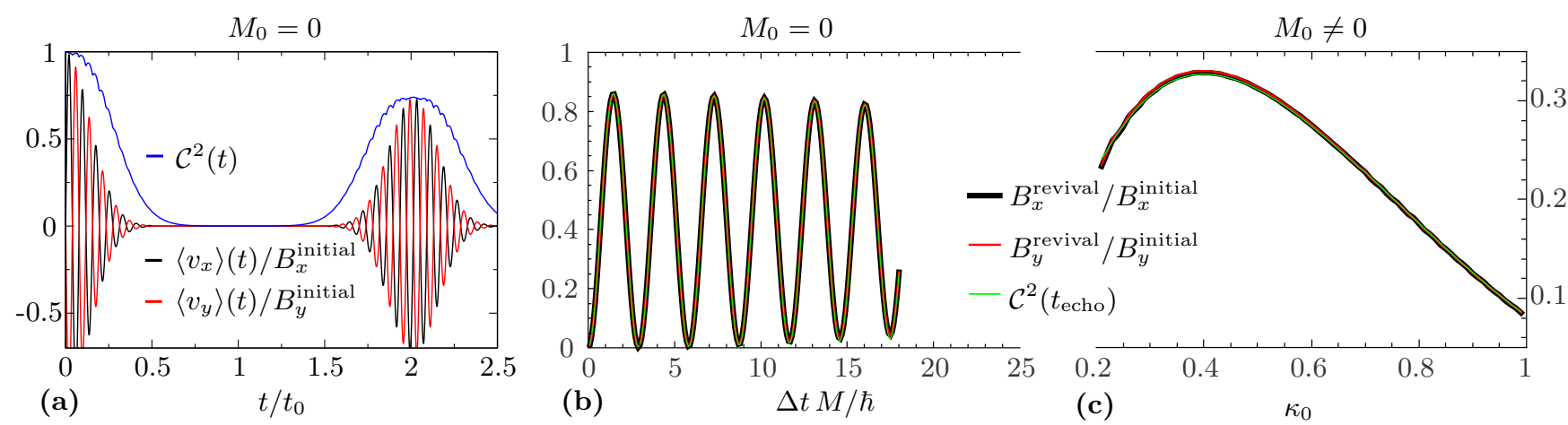

FIG. 4. Comparison between ZB echo simulations and analytical estimates for a gapless [panels (a), (b)] and a gapped [panel (c)] Dirac system. In panel (a), both the velocity expectation value and the correlation $\mathcal{C}^{2}(t)$, Eq. (28), are shown as functions of time, where $\mathcal{C}^{2}(t)$ and the $\mathrm{ZB}$ amplitude coincide at $t_{\text {echo }} \simeq 2 t_{0}$, in agreement with Eq. (38). Panel (b) compares the echo strength $\mathcal{C}\left(t_{\text {echo }}\right) \approx\left|A\left(\mathbf{k}_{0}, \Delta t\right)\right|($ see Ref. [29]), to the relative amplitude of the revived ZB both in the $x$ - and the $y$-directions as a function of $\Delta t$ for fixed $\kappa_{0}=\hbar v_{F} k_{0} / M=0.4$. The expected agreement can be seen. Panel (c) shows analogous data for a gapped Dirac system, where $\Delta t=1.4 \hbar / M$ whereas the mean wave vector of the wave packet, i.e., $\kappa_{0}$, is varied.

functions of $\Delta t$, i.e., of the pulse duration $\Delta t$. Numerically, the amplitude of the echo is obtained by searching for the largest difference between consecutive local maxima and minima, which are in a certain time interval around the expected echo time $t_{\text {echo }}=2 t_{0}+\Delta t, I=\left[t_{\text {echo }}-\tau, t_{\text {echo }}+\tau\right]$, to exclude the initial ZB from the automatic search in the data. We define the echo amplitude $B_{i}^{\text {revival }}$ of the ZB as

$$
B_{i}^{\text {revival }}=\max _{t \in I} \frac{\left|v_{i}^{\max }(t)-v_{i}^{\min }\left(t+\pi / \Omega_{\mathbf{k}_{0}}^{\mathrm{ZB}}\right)\right|}{2},
$$

where $v_{i}^{\max }$ is a local maximum and $v_{i}^{\min }$ is a local minimum of the velocity in direction $i$. Here, we use as interval width $\tau=$ $0.5 t_{0}$, but the exact value does not matter as long as the revival is included in the interval $I$ and the ZB that does not belong to the revival is excluded. Since no difference between $\mathcal{C}^{2}$ and the ratio between initial and revived amplitudes is visible in panel (b), the echo of the ZB has the expected strength, see Eq. (38).

In panel (c), a gapped Dirac system $\left(M_{0} \neq 0\right)$ is considered, and the echo strengths are shown for varying mean wavevectors $\kappa_{0}=\hbar v_{F} k_{0} / M$ and fixed $\Delta t=1.4 \hbar / M$. The relative amplitude of the revived ZB matches the quantum time mirror echo strength obtained by the correlation $\mathcal{C}^{2}\left(t_{\text {echo }}\right)$, confirming again our analytical expectations.

Hence, we have shown that the $\mathrm{ZB}$ echo behaves as the quantum time mirror in Ref. [30] where, for different band structures, the effects of position-dependent potentials in the Hamiltonian, such as disorder and electromagnetic fields are discussed additionally. To show that we recover the same results for the $\mathrm{ZB}$ echo also in these cases, we discuss the effect of disorder on the ZB echo in Appendix E. As expected, the relative echo strength decreases exponentially in time where the decay time is given by the elastic-scattering time.

Although we stay on the single-particle level, Rusin and Zawadzki [28] suggest that methods from nonlinear laser spectroscopy, such as the established two-photon echo [24] can be used to extract the ZB in a many-particle setup as has been performed for Bloch oscillations [25-27]. Most importantly, these suggested methods together with our proposed ways to prolongate the lifetime of the $\mathrm{ZB}$, could be sensitive enough to finally measure the ZB in solids.

\section{SUMMARY AND CONCLUSIONS}

The ZB as a promient hallmark of dynamics governed by the Dirac equation has so far resisted a direct measurement, although it is expected to exist in solid-state physics in systems, such as graphene, that can be described by an effective Dirac equation. We theoretically investigated different aspects of the dynamics of the driven $\mathrm{ZB}$ via analytical and numerical methods within a single-particle picture [41]. The basic concepts for understanding and describing the ZB in static Dirac systems were introduced in Sec. II where we also outlined our two strategies to counteract the ZB decay (that has hindered direct measurements) witj both strategies based on the time-dependent Hamiltonian (14).

The first one was discussed in Sec. III. A periodically driven mass term in (gapped) Dirac systems, see Eq. (15), was shown to give rise to a multimode $\mathrm{ZB}$. The latter longtime behavior reveals the existence of persistent $\mathrm{ZB}$ modes, emerging at frequencies stationary with respect to wavevector changes and not necessarily at simple multiples of the driving frequency. Such long-lived modes should allow for an experimental detection of the $\mathrm{ZB}$ much easier than standard rapidly decaying $\mathrm{ZB}$ modes.

The second strategy was dealt with in Sec. IV. The ZB revivals or echoes were shown to be generated via a protocol acting as a quantum time mirror $[29,30]$, i.e., requiring a short pulse opening temporarily a mass gap as defined in Eq. (27). The echo signal decays exponentially for weak disorder, i.e., behaves as expected in the presence of impurities or imperfections. Although ZB experiments remain challenging [20], it would be interesting to transfer established spin-echo-based protocols - mostly $T_{2}$-weighted imaging - to the ZB.

\section{ACKNOWLEDGMENTS}

We acknowledge support from Deutsche Forschungsgemeinschaft (DFG, German Research Foundation) through 
Projects No. 10314695032 (CRC 1277, Subproject No. A07) and No. GRK 1570.

\section{APPENDIX A: RELATION BETWEEN $A_{ \pm}$AND $B_{ \pm}$AND THE INITIAL OCCUPATIONS OF A GIVEN WAVE PACKET}

This Appendix shows the relation between the amplitudes of a given initial state and the quantities $A_{ \pm}$and $B_{ \pm}$which are used to denote the amplitudes of the ZB in the RWA and HDF, respectively.

\section{1. $A_{ \pm}$in the rotating-wave approximation}

In the RWA, the ansatz used to solve the transformed Dirac equation reads

$$
\psi_{\mathbf{k}}^{\mathrm{RWA}}(t)=\left(\begin{array}{c}
A_{+} e^{-i\left[\left(\omega_{D}+\omega_{R}\right) / 2\right] t}+A_{-} e^{-i\left[\left(\omega_{D}-\omega_{R}\right) / 2\right] t} \\
C_{+} e^{i\left[\left(\omega_{D}+\omega_{R}\right) / 2\right] t}+C_{-} e^{i\left[\left(\omega_{D}-\omega_{R}\right) / 2\right] t}
\end{array}\right),
$$

with

$$
C_{ \pm}=A_{ \pm} \frac{\hbar \sqrt{1+\kappa^{2}}}{\tilde{M} \kappa} e^{i \gamma_{\mathbf{k}}}\left(\Delta \pm \omega_{R}\right)
$$

and, thus, for $t=0$,

$$
\psi_{\mathbf{k}}^{\mathrm{RWA}}(0)=\left(\begin{array}{c}
A_{+}+A_{-} \\
\frac{\hbar \sqrt{1+\kappa^{2}}}{\tilde{M} \kappa} e^{i \gamma_{\mathbf{k}}}\left[A_{+}\left(\Delta+\omega_{R}\right)+A_{-}\left(\Delta-\omega_{R}\right)\right]
\end{array}\right) .
$$

Note that $\psi_{\mathbf{k}}^{\mathrm{RWA}}$ solves the Dirac equation obtained after rotating the spin degree of freedom such that the static Hamiltonian is diagonal. In other words, to compare $A_{ \pm}$with the amplitudes of any initial state,

$$
\phi_{\mathbf{k}}^{0}=\left(\begin{array}{c}
\alpha_{\mathbf{k}} \\
\beta_{\mathbf{k}}
\end{array}\right),
$$

the transformation $S^{\mathrm{RWA}}$ is introduced through

$$
S^{\mathrm{RWA}} \phi_{\mathbf{k}}^{0}=\psi_{\mathbf{k}}^{\mathrm{RWA}}(0) \text {. }
$$

For the Hamiltonian in Eq. (14), the transformation is

$$
S^{\mathrm{RWA}}=e^{-i\left(\vartheta_{\mathbf{k}} / 2\right) \mathbf{m}_{\mathbf{k}} \cdot \boldsymbol{\sigma}},
$$

where $\mathbf{m}_{\mathbf{k}}=\left(k_{y},-k_{x}, 0\right)$ is the rotation axis and the amount of rotation $\vartheta_{\mathbf{k}}=\left(\frac{\pi}{2}-\arctan ^{-1} \frac{M_{0}}{\hbar v_{F} k}\right)$ is equal to the polar angle in the Bloch sphere.

\section{2. $B_{ \pm}$at high-driving frequency}

In the HDF limit, the ansatz that is used to solve the Dirac equation is

$\psi_{\mathbf{k}}^{\mathrm{HDF}}(t)=\left(\begin{array}{c}{\left[B_{+} e^{i} \Omega_{\mathbf{k}}^{\mathrm{st}} t / 2+B_{-} e^{-i \Omega_{\mathbf{k}}^{\mathrm{st}} t / 2}\right] e^{-i\left(\tilde{M} / \hbar \omega_{D}\right) \sin \left(\omega_{D} t\right)}} \\ {\left[D_{+} e^{i} \Omega_{\mathbf{k}}^{\mathrm{st}} t / 2+D_{-} e^{-i \Omega_{\mathbf{k}}^{\mathrm{st}} t / 2}\right] e^{+i\left(\tilde{M} / \hbar \omega_{D}\right) \sin \left(\omega_{D} t\right)}}\end{array}\right)$,

with

$$
D_{ \pm}=-B_{ \pm} \frac{e^{i \gamma_{\mathbf{k}}}}{2 v_{F} k}\left(2 \frac{M_{0}}{\hbar} \pm \Omega_{\mathbf{k}}^{\mathrm{st}}\right)
$$

and, thus, for $t=0$,

$$
\psi_{\mathbf{k}}^{\mathrm{HDF}}(0)=\left(\begin{array}{c}
B_{+}+B_{-} \\
-\frac{e^{i\rangle_{\mathbf{k}}}}{2 v_{F} k}\left[B_{+}\left(2 \frac{M_{0}}{\hbar} \pm \Omega_{\mathbf{k}}^{\mathrm{st}}\right)+B_{-}\left(2 \frac{M_{0}}{\hbar} \pm \Omega_{\mathbf{k}}^{\mathrm{st}}\right)\right]
\end{array}\right) .
$$

Since there is no transformation used in the considered Hamiltonian for the HDF case, we can directly compare $B_{ \pm}$with the amplitudes of any initial state of the form of Eq. (A4). In contrast to the RWA, we directly obtain

$$
\phi_{\mathbf{k}}^{0}=\psi_{\mathbf{k}}^{\mathrm{HDF}}(0)
$$

\section{APPENDIX B: EMERGENCE OF HIGHER $\omega_{D}$ MODES IN THE DRIVEN ZITTERBEWEGUNG}

This Appendix is supposed to motivate qualitatively why for larger driving amplitudes, $\tilde{M}$, higher-order frequencies $n \omega_{D} \pm \omega_{R}$ of the ZB appear instead of only the ZB frequencies predicted by the RWA $\omega_{D} \pm \omega_{R}$. In the following, we use the static energy eigenstates $\left|\varphi_{\mathbf{k}, \pm}\right\rangle$ as a basis. Any plane wave at $\mathbf{k}$ can be written in that basis similar to the static case in Eq. (6),

$$
\left|\psi_{\mathbf{k}}(t)\right\rangle=c_{\mathbf{k}}^{+}(t) e^{-i \omega_{\mathbf{k},+} t}\left|\varphi_{\mathbf{k},+}\right\rangle+c_{\mathbf{k}}^{-}(t) e^{-i \omega_{\mathbf{k},-} t}\left|\varphi_{\mathbf{k},-}\right\rangle,
$$

with the only difference that the occupations $c_{\mathbf{k}, \pm}(t)$ change over time. The off-diagonal part of the velocity operator is then

$$
\left\langle\mathbf{v}^{\mathrm{ZB}}\right\rangle_{\mathbf{k}}(t)=2 \operatorname{Re}\left\{c_{+, \mathbf{k}}(t) c_{-, \mathbf{k}}^{*}(t) e^{-i \Omega_{\mathbf{k}}^{\mathrm{st} t}}\left\langle\varphi_{\mathbf{k},-}|\mathbf{v}| \varphi_{\mathbf{k},+}\right\rangle\right\} .
$$

By definition, the time-dependent occupations can be written as

$$
c_{\mathbf{k}, \pm}(t)=\left\langle\varphi_{\mathbf{k}, \pm}\left|U_{I}(t, 0)\right| \psi_{\mathbf{k}}(t=0)\right\rangle,
$$

with the help of the time-evolution operator in the interaction picture $U_{I}$, which can be expressed in terms of a Dyson series [42],

$$
\begin{aligned}
U_{I}(t)= & 1-\frac{i}{\hbar} \int_{0}^{t} V_{I}\left(t^{\prime}\right) d t^{\prime}+\left(-\frac{i}{\hbar}\right)^{2} \int_{0}^{t} d t^{\prime} \\
& \times \int_{0}^{t^{\prime}} d t^{\prime \prime} V_{I}\left(t^{\prime}\right) V_{I}\left(t^{\prime \prime}\right)+\cdots
\end{aligned}
$$

With a harmonic driving $V_{I} \propto e^{i \omega_{D} t}$ as used in Sec. III, the first order of the occupations $c_{\mathbf{k}, \pm}$ contains terms with the same frequency $\omega_{D}$. In order $n$, however, we will get $e^{i n \omega_{D} t}$ terms of the occupations, which will be reflected in the velocity, see Eq. (B2). Thus, for larger driving amplitudes, higher-order oscillations in $\omega_{D}$ are expected.

\section{APPENDIX C: ADDITIONAL LONG-TIME ZB MODES}

In this Appendix, we discuss numerically the additional (partly) surviving modes shown in Figs. 2(c) and 2(d). There, the modes with $\omega=n \omega_{D}$ survive as discussed in the main text, but also the other modes close to the crossings with the horizontal lines $\omega=n \omega_{D}$. Since these are located in regions not explained by our analytical approximations ( $\tilde{M}$ is too large), we can investigate their $\mathbf{k}$ dependence-and, thus, whether they dephase or not over the wave-packet's widthonly numerically. To this end, we simulate the propagation of wave packets with different initial energies for a gapless Dirac cone $\left(M_{0}=0\right)$ because the effect can be better seen there. So far, the propagated Gaussian wave packet is centered around $\mathbf{k}_{0}=\left(0.4 M_{0} / \hbar v_{F}, 0\right)^{T}$ with a $\mathbf{k}$ space width of $\Delta k=k_{0} / 10$. To analyze the $\mathbf{k}$ dependence of the ZB modes, now, we simulate two wave packets centered around $\langle\mathbf{k}\rangle=\mathbf{k}_{0} \pm(\Delta k, 0)^{T}$ instead. If the positions of the modes do not change in the 


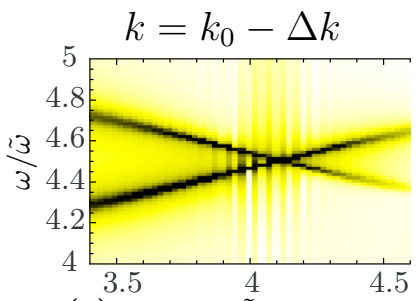

(a)
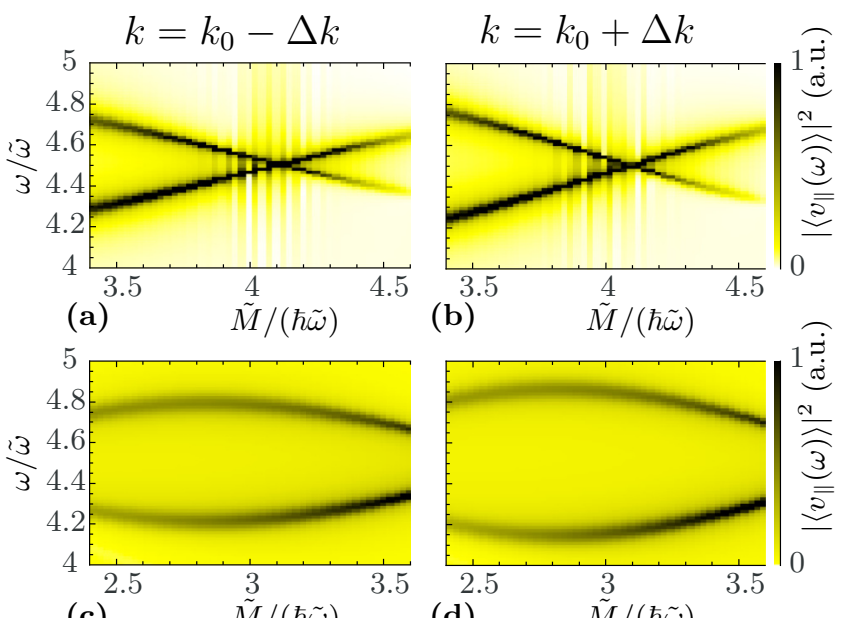

(c) $\tilde{M} /(\hbar \tilde{\omega})$ (b)

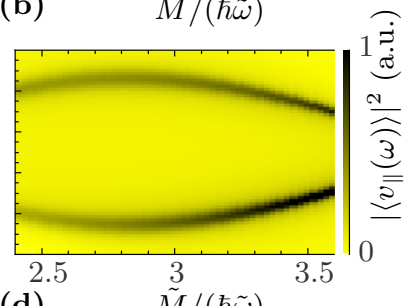

(d)
¿

FIG. 5. Analysis explaining why the modes at the $\operatorname{crossing}(\omega \approx$ $\left.n \omega_{D}\right)$ survive in Fig. 2(d). Here, the parallel velocity $\left|\left\langle v_{\|}(\omega)\right\rangle\right|^{2}$ is shown for $M_{0}=0$ as a function of $\tilde{M}$ for $\omega_{D}=1.5 \tilde{\omega}$ with the normalization frequency $\tilde{\omega}=2.5 v_{F} k_{0}$ for two different energies with $k=0.36 \tilde{\omega} / v_{F}$ [in (a) and (c)] and $k=0.44 \tilde{\omega} / v_{F}$ [in (b) and (d)]. The frequency of the $\mathrm{ZB}$ is quite independent at the crossings, i.e., (a) and (b) are very similar, whereas away from the crossing, it is energy dependent, i.e., (c) and (d) differ. Since only energy-independent modes survive as discussed in the main text, only modes at the crossings can be seen after at late times [Fig. 2(d)].

diagrams, they are not $\mathbf{k}$ dependent (at least, for the relevant modes in the wave packet) and are supposed to survive. On the other hand, if the frequencies of the modes do change, they are $\mathbf{k}$ dependent and should, therefore, dephase. In Fig. 5, the data of these additional simulations are shown, i.e., wave packets of different initial energies are propagated in a much smaller parameter regime than before. Here, the fixed parameters are $M_{0}=0$ and $\omega_{D}=1.5 \tilde{\omega}$ with the unit frequency $\tilde{\omega}=2.5 v_{F} k_{0}$. Panels (a) and (b) show the ZB modes at the crossing of the horizontal line $\omega=4.5 \tilde{\omega}=3 \omega_{D}$ for different initial energies, whereas panels (c) and (d) depict the region away from those crossings. Since the position of the crossing at $\tilde{M} \approx 4.1 \hbar \tilde{\omega}$ does not considerably change, the crossings are rather $\mathbf{k}$ independent over the width of the wave packet. On the other hand, the frequency change in the modes away from the crossing is visible with the bare eye since there the $\mathbf{k}$ dependence is much stronger. Consequently, only the modes around the crossings are supposed to persist as is shown in the long-time behavior where modes in the other regions decay over time.

\section{APPENDIX D: PHASE INFLUENCE OF THE ZB ECHO}

This Appendix deals with the rather technical point concerning the phase change in the velocity matrix element $v_{\mathbf{k}}=\left\langle\varphi_{\mathbf{k},+}\left|v_{i}\right| \varphi_{\mathbf{k},-}\right\rangle$ due to the pulse in Eq. (35). For gapless Dirac systems, the complex number $\left\langle\varphi_{\mathbf{k},-}\left|v_{i}\right| \varphi_{\mathbf{k},+}\right\rangle$ is purely imaginary leading to a constant phase jump of $\pi$ due to $\left\langle\varphi_{\mathbf{k},+}\left|v_{i}\right| \varphi_{\mathbf{k},-}\right\rangle=\left\langle\varphi_{\mathbf{k},-}\left|v_{i}\right| \varphi_{\mathbf{k},+}\right\rangle^{*}$ which does not effect the amplitude of the echo. For a general system, on the other hand, this phase change might be $\mathbf{k}$ dependent, implying possibly an altered interference of the modes in a wave packet [compare Eq. (33)]. However, this should, in general, not

lead to a further reduction of the $\mathrm{ZB}$ compared to the initial $\mathrm{ZB}$-as long as this phase $v_{\mathbf{k}}$ is unrelated to the phase of $\alpha_{\mathbf{k}}=c_{\mathbf{k}}^{+}\left(c_{\mathbf{k}}^{-}\right)^{*}$. In this case, the interference of different $\mathbf{k}$ modes at the start and at the echo time is expected to be qualitatively the same.

An exception would be the case $\nu_{\mathbf{k}}=\alpha_{\mathbf{k}}$ for all $\mathbf{k}$. Note that this is highly unlikely because these two quantities are independent $-\alpha_{\mathbf{k}}$ depends only on the initial wave packet, whereas $v_{\mathbf{k}}$ only depends on unperturbed Hamiltonian and the velocity operator. In this unrealistic correlated case, the $\mathrm{ZB}$ at $t=0$ would be diminished because of nonperfect interference of different $\mathbf{k}$ modes. On the other hand, the $\mathrm{ZB}$ at $t_{1}=t_{0}$ would be enhanced since $\alpha_{\mathbf{k}}$ and $-v_{\mathbf{k}}$ would exactly cancel for all $\mathbf{k}$ leading to perfect interference of all modes. Thus, for a transition amplitude close to one $(|A(\mathbf{k}, \Delta t)| \lesssim 1)$, the amplitude of the echo $\mathrm{ZB}$ could be even higher than of the initial ZB. However, we do not consider this highly unlikely case further in this paper. Instead, we neglect the effect of $v_{\mathbf{k}}$, which is justified for narrow wave packets in $\mathbf{k}$ space such that $v_{\mathbf{k}} \simeq v_{\mathbf{k}_{0}}$.

\section{APPENDIX E: EFFECT OF DISORDER ON THE ZB ECHO}

In the main text, we showed that the echo of $\mathrm{ZB}$ induced by our QTM behaves as expected in pristine and gapped Dirac systems. Now, we could continue and verify all other results obtained in the general paper about QTMs [30], such as asymmetric band structures and position-dependent potentials in the Hamiltonian. There is no reason for the results to differ from the QTM case, and, hence, why we only show exemplarily the effect of disorder discussed in Refs. [29,30]. The previous results show that disorder cannot be effectively time inverted by the QTM and leads to an exponential decay of the echo strength (measured by the echo fidelity) as a function of propagation time, which is, in the present case, the echo time $t_{\text {echo }} \simeq 2 t_{0}$. Due to the overlap of states with positive and negative energies in the $\mathrm{ZB}$, the relative amplitude of the velocity is closely related to the echo fidelity, and we expect a similar behavior, i.e., an exponential decay.

To compare with the previous results, we use the same setup as in Ref. [29], i.e., pristine graphene with a pulse that opens a mass gap of strength $\tilde{M}=M$ and a pseudo-spinindependent impurity potential $V_{\mathrm{imp}}(\mathbf{r})$. The only difference compared to Ref. [29] is that, in order to generate the ZB, the initial wave packet lives in both bands.

In order to generate the random inhomogeneous disorder potential $V_{\text {imp }}$, every grid point is assigned a normal distributed random number $\beta_{i}$. To avoid a highly fluctuating potential, an average over neighboring points weighted by a Gaussian profile with a range $l_{0}$, is taken at each site,

$$
V_{\text {imp }}(\mathbf{r})=\frac{u_{0}}{\mathcal{N}} \sum_{i=1}^{N} \beta_{i} e^{-\left[\left(\mathbf{r}-\mathbf{r}_{i}\right)^{2} / l_{0}^{2}\right]}
$$

Here, the sum runs over grid points, $u_{0}$ is related to the mean impurity strength, and $\mathcal{N}$ is a normalization factor to account for different realizations with the same parameters $u_{0}$ and $l_{0}$,

$$
\mathcal{N}=\left[\frac{1}{A} \int_{A} d^{2} r\left(\sum_{i=1}^{N} \beta_{i} e^{-\left[\left(\mathbf{r}-\mathbf{r}_{i}\right)^{2} / l_{0}^{2}\right]}\right)^{2}\right]^{1 / 2},
$$



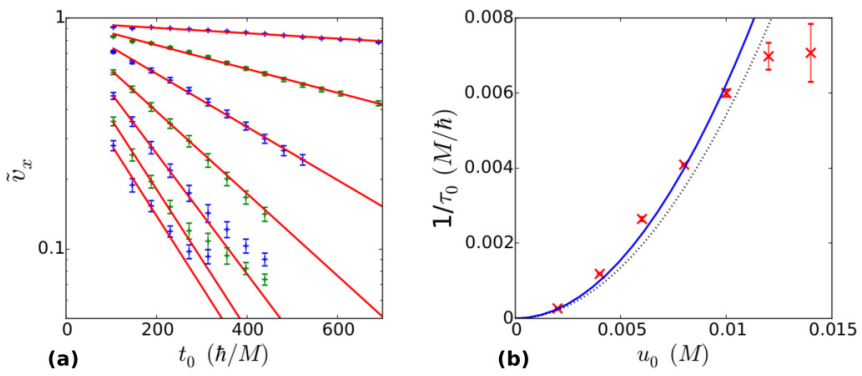

FIG. 6. ZB echoes in the presence of disorder. (a) The timedependent revival strength $\tilde{v}_{x}=B_{x}^{\text {revival }} / B_{x}^{\text {initial }}$ is shown as a function of the pulse time $t_{0}$ for different disorder strengths ranging from $u_{0}=0.002 M$ to $0.014 M$. An exponential decay can be seen in the weak disorder regime. (b) The decay rate $1 / \tau_{0}$ is extracted by a fit [red lines in (a)] and plotted as a function of $u_{0}$ and compared to the analytically expected scattering time (black dotted) from Eq. (E3). The quadratic fit in $u_{0}$ (blue line) to the data points is close to the expected scattering time. Saturation is obtained for larger $u_{0}$. For more details, see the discussion of disorder in Ref. [29].

where $A$ is the finite area of the grid. $\mathcal{N}$ can be thought of the mean deviation of the potential strength over the whole area.
The elastic-scattering time $\tau_{0}$ linked to $V_{\mathrm{imp}}(\mathbf{r})$ is given by (see, e.g., Ref. [43]),

$$
\frac{\hbar}{\tau_{0}}=\frac{2 \pi}{v_{F} \hbar} k u_{0}^{2} l_{0}^{2} \exp \left(-k^{2} l_{0}^{2}\right) I_{0}\left(k^{2} l_{0}^{2}\right),
$$

where $I_{0}$ is the modified Bessel function of the 0 th kind.

As before, we measure the revival of the $\mathrm{ZB}$ through the relative echo amplitude, $B_{i}^{\text {revival }} / B_{i}^{\text {initial }}$, which is plotted as a function of the pulse time $t_{0}$ for different disorder strengths $u_{0}$ in Fig. 6(a). Indeed, we see exponential decays of the echo as function of the pulse time $t_{0}$ where again a saturation is achieved for high $u_{0}$ and $t_{0}$, in analogy to Ref. [29]. The fitted decay rates $1 / \tau_{0}$ of panel (a) (red lines) are plotted in panel (b) as a function of the disorder strength $u_{0}$, which are expected to increase quadratically [compare Eq. (E3)]. Up to some value of $u_{0} \simeq 0.012 M$, the decay rate, indeed, increases quadratically as can be seen by the quadratic fit (blue) and is slightly larger but close enough to the purely analytically expected decay (black dotted line). Above $u_{0} \simeq$ $0.012 M$, deviations are expected [29] since the golden-ruledecay regime is no longer valid, which was used to derive $\tau_{0}$ in Eq. (E3).
[1] E. Schrödinger, Sitz. Press. Akad. Wiss. Phys.-Math. 24, 418 (1930).

[2] W. Zawadzki and T. M. Rusin, J. Phys.: Condens. Matter 23, 143201 (2011).

[3] K. Huang, Am. J. Phys. 20, 479 (1952).

[4] J. Schliemann, D. Loss, and R. M. Westervelt, Phys. Rev. Lett. 94, 206801 (2005).

[5] J. Schliemann, D. Loss, and R. M. Westervelt, Phys. Rev. B 73, 085323 (2006).

[6] W. Zawadzki and T. M. Rusin, Phys. Lett. A 374, 3533 (2010).

[7] W. Zawadzki, Phys. Rev. B 74, 205439 (2006).

[8] M. I. Katsnelson, Eur. Phys. J. B 51, 157 (2006).

[9] T. M. Rusin and W. Zawadzki, Phys. Rev. B 78, 125419 (2008).

[10] L.-K. Shi, S.-C. Zhang, and K. Chang, Phys. Rev. B 87, 161115(R) (2013).

[11] G. J. Ferreira, R. P. Maciel, P. H. Penteado, and J. C. Egues, Phys. Rev. B 98, 165120 (2018).

[12] W. Wang, C. Gu, Y. Zhou, and H. Fangohr, Phys. Rev. B 96, 024430 (2017).

[13] E. S. Sedov, Y. G. Rubo, and A. V. Kavokin, Phys. Rev. B 97, 245312 (2018).

[14] J. Schliemann, New J. Phys. 10, 043024 (2008).

[15] J. Schliemann, Phys. Rev. B 77, 125303 (2008).

[16] R. Gerritsma, G. Kirchmair, F. Zahringer, E. Solano, R. Blatt, and C. F. Roos, Nature (London) 463, 68 (2010).

[17] C. Qu, C. Hamner, M. Gong, C. Zhang, and P. Engels, Phys. Rev. A 88, 021604(R) (2013).

[18] L. J. LeBlanc, M. C. Beeler, K. Jiménez-García, A. R. Perry, S. Sugawa, R. A. Williams, and I. B. Spielman, New J. Phys. 15, 073011 (2013).

[19] C. Wang, C. Gao, C.-M. Jian, and H. Zhai, Phys. Rev. Lett. 105, 160403 (2010)
[20] I. Stepanov, M. Ersfeld, A. V. Poshakinskiy, M. Lepsa, E. L. Ivchenko, S. A. Tarasenko, and B. Beschoten, arXiv:1612.06190.

[21] Y. Iwasaki, Y. Hashimoto, T. Nakamura, and S. Katsumoto, J. Phys.: Conf. Ser. 864, 012054 (2017).

[22] T. M. Rusin and W. Zawadzki, Phys. Rev. B 88, 235404 (2013).

[23] C. S. Ho, M. B. A. Jalil, and S. G. Tan, Europhys. Lett. 108, 27012 (2014).

[24] R. Boyd, Nonlinear Optics (Academic, San Diego, 2003).

[25] G. von Plessen and P. Thomas, Phys. Rev. B 45, 9185 (1992).

[26] S. Mukamel, Principles of Nonlinear Molecular Spectroscopy (Oxford University Press, New York, 1995).

[27] J. Feldmann, K. Leo, J. Shah, D. A. B. Miller, J. E. Cunningham, T. Meier, G. von Plessen, A. Schulze, P. Thomas, and S. Schmitt-Rink, Phys. Rev. B 46, 7252 (1992).

[28] T. M. Rusin and W. Zawadzki, Semicond. Sci. Technol. 29, 125010 (2014).

[29] P. Reck, C. Gorini, A. Goussev, V. Krueckl, M. Fink, and K. Richter, Phys. Rev. B 95, 165421 (2017).

[30] P. Reck, C. Gorini, and K. Richter, Phys. Rev. B 98, 125421 (2018).

[31] J. A. Lock, Am. J. Phys. 47, 797 (1979).

[32] T. M. Rusin and W. Zawadzki, Phys. Rev. B 76, 195439 (2007).

[33] Further relaxation processes can be added phenomenologically as performed e.g., in Ref. [28].

[34] V. Krückl, Wave packets in mesoscopic systems: From timedependent dynamics to transport phenomena in graphene and topological insulators, Ph.D. thesis, Universität Regensburg, 2013, the basic version of the algorithm is available at TQT Home, http://www.krueckl.de/\#en/tqt.php.

[35] P. Reck, Quantum Echoes and revivals in two-band systems and Bose-Einstein condensates, Ph.D. thesis, Universität Regensburg, 2018. 
[36] G. Auletta, M. Fortunato, and G. Parisi, Quantum Mechanics (Cambridge University Press, Cambridge, UK, 2009).

[37] This is very likely due to the fact that Ref. [22] considers pristine graphene where no parallel $\mathrm{ZB}$ is expected in the static case.

[38] C. Lanczos, J. Res. Natl. Bur. Stand. 45, 255 (1950).

[39] P. Reck, C. Gorini, A. Goussev, V. Krueckl, M. Fink, and K. Richter, New J. Phys. 20, 033013 (2018).

[40] At $t_{\text {echo }}$, the matrix element of the velocity operator in Eqs. (34) and (35) reads $\left\langle\varphi_{\mathbf{k},+}\left|v_{i}\right| \varphi_{\mathbf{k},-}\right\rangle=\left\langle\varphi_{\mathbf{k},-}\left|v_{i}\right| \varphi_{\mathbf{k},+}\right\rangle^{*}$, i.e., the phase changes sign. For gapless Dirac systems, the matrix element $\left\langle\varphi_{\mathbf{k},-}|\mathbf{v}| \varphi_{\mathbf{k},+}\right\rangle$ is purely imaginary. This gives rise to a $\pi$-phase jump, irrelevant for the $\mathrm{ZB}$ amplitude. However, the phase change is, in general, $\mathbf{k}$ dependent, and its effect is discussed in Appendix D but neglected henceforth.

[41] Numerous techniques are available to describe relaxation.

[42] J. Sakurai and J. Napolitano, Modern Quantum Mechanics (Pearson, London, 2011).

[43] E. Akkermans and G. Montambaux, Mesoscopic Physics of Electrons and Photons (Cambridge University Press, Cambridge, UK, 2007). 